\title{
Identification of Chemotaxis Sensory Proteins for Amino Acids in Pseudomonas fluorescens Pf0-1 and Their Involvement in Chemotaxis to Tomato Root Exudate and Root Colonization
}

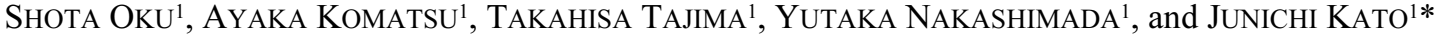 \\ ${ }^{1}$ Department of Molecular Biotechnology, Graduate School of Advanced Sciences of Matter, Hiroshima University, \\ 1-3-1 Kagamiyama, Higashi-Hiroshima, Hiroshima 739-8530, Japan
}

(Received January 16, 2012-Accepted May 16, 2012_Published online September 5, 2012)

Pseudomonas fluorescens Pf0-1 showed positive chemotactic responses toward 20 commonly-occurring L-amino acids. Genomic analysis revealed that P. fluorescens Pf0-1 possesses three genes (Pf101_0124, Pf101_0354, and Pf101_4431) homologous to the Pseudomonas aeruginosa PAO1 pctA gene, which has been identified as a chemotaxis sensory protein for amino acids. When Pf01_4431, Pf101_0124, and Pfl01_0354 were introduced into the pctA pctB pct $C$ triple mutant of $P$. aeruginosa PAO1, a mutant defective in chemotaxis to amino acids, its transformants showed chemotactic responses to 18, 16, and one amino acid, respectively. This result suggests that Pf01_4431, Pfl01_0124, and Pf101_0354 are chemotaxis sensory proteins for amino acids and their genes were designated $\operatorname{cta} A, \operatorname{ctaB}$, and $c t a C$, respectively. The ctaA ctaB ctaC triple mutant of P. fluorescens Pf0-1 showed only weak responses to Cys and Pro but no responses to the other 18 amino acids, indicating that $\mathrm{CtaA}, \mathrm{CtaB}$, and $\mathrm{CtaC}$ are major chemotaxis sensory proteins in $P$. fluorescens Pf0-1. Tomato root colonization by $P$. fluorescens strains was analyzed by gnotobiotic competitive root colonization assay. It was found that ctaA ctaB ctaC mutant was less competitive than the wild-type strain, suggesting that chemotaxis to amino acids, major components of root exudate, has an important role in root colonization by $P$. fluorescens Pf0-1. The ctaA ctaB ctaC triple mutant was more competitive than the cheA mutant of $P$. fluorescens Pf0-1, which is non-chemotactic, but motile. This result suggests that chemoattractants other than amino acids are also involved in root colonization by $P$. fluorescens Pf0-1.

Key words: plant growth-promoting rhizobacteria, chemotaxis, methyl-accepting chemotaxis protein, Pseudomonas fluorescens, root colonization

Certain strains of Pseudomonas fluorescens exert beneficial effects on plants $(3,13,17)$. These strains are able to enhance plant growth indirectly by preventing the growth or action of plant-pathogenic microorganisms. P. fluorescens F113 protects sugar beet seedlings from damping-off disease caused by the fungal pathogen Pythium ultimum by producing the antifungal metabolite 2,4-diacetylphloroglucinol, hydrogen cyanide, and extracellular protease $(1,22)$. P. fluorescens strain $2-79$ is able to suppress wheat take-all produced by Gaeumannomyces graminis var. tritici by means of producing antibiotics phenazine-1-carboxylic acid $(32,33)$. $P$. fluorescens WCS365 is a biocontrol agent against Fusarium oxysporum, which causes tomato foot and root rot (4). This strain is an efficient root colonizer and it is assumed that it can favorably compete for available habitable niches on a root surface with the pathogenic fungus.

Efficient root colonization by plant-beneficial rhizobacteria is assumed to be essential for the biocontrol of root pathogens (30). In previous studies, it was demonstrated that motility and chemotaxis are important traits for root colonization by $P$. fluorescens. Simons et al. reported that a mutant of $P$. fluorescens WCS365, lacking flagella was outcompeted in the root-tip colonization assay when inoculated in competition with the parental WCS365 strain (24). Conversely, Barahona

\footnotetext{
* Corresponding author. E-mail: jun@hiroshima-u.ac.jp;

Tel: +81-82-424-7757; Fax: +81-82-424-7047.
}

et al. showed that a hypermotile mutant of $P$. fluorescens F113, was more competitive for rhizosphere colonization than the wild-type strain and exhibited improved biocontrol activity against the pathogenic fungus $F$. oxysporum and the pathogenic oomycete Phytophthora cactorum compared with the wild type strain (3). de Weert et al. demonstrated that cheA mutant of $P$. fluorescens WCS365, which is nonchemotactic but motile, colonized the tomato root tip less efficiently than the wild-type strain in the competitive root colonization assay (6).

Plant root exudates contain various organic compounds. Major components of tomato root exudate are amino acids (glutamic acid, aspartic acid, leucine, isoleucine, and lysine as major components [25]), organic acids (especially citric acid, malic acid and succinic acid [10]), and sugars (glucose and xylose as major components [14]). Previous studies demonstrated that $P$. fluorescens strains exhibit chemotactic responses toward plant seed and root exudates and their components $(6,20,26,30)$; therefore, it is supposed that chemotaxis to components of plant root exudates is involved in effective root colonization.

Methyl-accepting chemotaxis proteins (MCPs) are chemotaxis sensory proteins responsible for the detection of chemotactic ligands (11). MCPs are membrane-spanning homodimers and typical features of MCPs are as follows: a positively charged $\mathrm{N}$ terminus followed by a hydrophobic membrane-spanning region, a hydrophilic periplasmic 
domain, a second hydrophobic membrane-spanning region and a hydrophilic cytoplasmic domain (7). Chemotactic ligands bind to periplasmic domains of MCPs and their binding initiates chemotaxis signaling. The diverse ligand specificities among MCPs reflect amino acid sequence diversities of periplasmic domains of MCPs. The C-terminal cytoplasmic domains of MCPs are relatively conserved. A 44-amino-acid highly conserved domain (HCD) is located in the cytoplasmic domain. MCPs from phylogenetically diverse bacteria have been shown to possess HCD (34). Blastp analysis found that in P. fluorescens Pf0-1 (accession number: CP000094), Pf5 (accession number: CP000076), and SBW25 (accession number: AM181176) genomes, there are 37, 34, and 46 gene products possessing HCD, respectively, and all have been annotated as MCPs; however, it is still unknown which exudate components and MCPs are involved in efficient root colonization by $P$. fluorescens. In the present study, we identified and characterized MCPs for amino acids, major components of plant root exudates, in P. fluorescens Pf0-1 and investigated their involvement in chemotaxis toward tomato root exudate and root colonization.

\section{Materials and Methods}

Bacterial strains, plasmids, and growth conditions

Bacterial strains and plasmids used in this study are listed in Table 1. Escherichia coli JM109 (18) and S17-1 (23) were used for plasmid construction and transconjugation, respectively. $P$. fluorescens, Pseudomonas aeruginosa and E. coli strains were grown with shaking in $2 \times$ YT medium (18) supplemented with appropriate antibiotics. $P$. aeruginosa and $E$. coli strains were cultivated at $37^{\circ} \mathrm{C}$, while $P$. fluorescens strains were grown at $28^{\circ} \mathrm{C}$.

\section{Chemotaxis assay}

The computer-assisted capillary assay method was carried out as described previously (16). Cells in a $10 \mu \mathrm{L}$ suspension were placed on a coverslip, and the assay was started by placing the coverslip upside down on the U-shaped spacer to fill the chemotaxis chamber with the cell suspension. Cells were videotaped over 3 min. Digital image processing was used to count the number of bacteria accumulating toward the mouth of a capillary containing a known concentration of an attractant plus $1 \%(\mathrm{w} / \mathrm{v})$ agarose. The strength of the chemotactic response was determined by the number of bacterial cell per frame. The chemotaxis buffer was 10 mM HEPES ( $N$-2-hydroxyethylpiperazine- $N$ '-ethanesulfonic acid) buffer $(\mathrm{pH}$ 7.0).

\section{DNA manipulation}

Standard procedures were used for plasmid DNA preparations, restriction enzyme digestions, ligations, transformations, and agarose gel electrophoresis (18). PCR reactions were carried out using KOD Plus DNA polymerase (Toyobo, Tokyo, Japan) according to the manufacturer's instructions. Oligonucleotides used for PCR are listed in Table 2. P. aeruginosa was transformed by electroporation as described previously (15). Plasmids were introduced to $P$. fluorescens strains by transconjugation using $E$. coli S17-1 (23).

\section{Plasmid construction and construction of deletion mutants of P. fluorescens $P f O-1$}

The Pf101_0124, Pf101_0354, and Pf101_4431 genes were amplified from $P$. fluorescens Pf0-1 genome by PCR using PFL01f/

Table 1. Bacterial strains and plasmids used in this study

\begin{tabular}{|c|c|c|c|}
\hline Strain or plasmid & \multicolumn{2}{|c|}{ Relevant characteristics } & Reference or source \\
\hline \multicolumn{4}{|l|}{ Pseudomonas fluorescens } \\
\hline Pf0-1 & wild-type strain & & 5 \\
\hline FL0124 & Pf0-1 derivative, $\triangle$ ctaA (Pf101_4431) & $\triangle c t a C\left(P f 101 \_0354\right)$ & This study \\
\hline FL0354 & Pf0-1 derivative, $\triangle$ ctaA (Pf101_4431) & $\triangle c t a B(\mathrm{Pfl01} 0124)$ & This study \\
\hline FL4431 & Pf0-1 derivative, $\triangle c t a B$ (Pf101_0124) & $\triangle c t a C(\mathrm{Pf101} 0354)$ & This study \\
\hline FLD3 & Pf0-1 derivative, $\triangle c t a A \Delta c t a B \triangle c t a C$ & & This study \\
\hline Pf0-1 $1 \Delta$ cheA & Pf0-1 derivative, $\Delta$ cheA (Pf101_2038) & & This study \\
\hline Pf01Rif & Pf0-1 derivative, spontaneous rifampici & sistant mutant & This study \\
\hline FLD3Rif & FLD3 derivative, spontaneous rifampic & esistant mutant, $\triangle c t a A \triangle c t a B \triangle c t a C$ & This study \\
\hline \multicolumn{4}{|l|}{ Pseudomonas aeruginosa } \\
\hline PAO1 & wild-type strain & & 9 \\
\hline PCT2 & PAO1 derivative, $\triangle p c t A \Delta p c t B \Delta p c t C$ & & 31 \\
\hline \multicolumn{4}{|l|}{ Escherichia coli } \\
\hline JM109 & $\begin{array}{l}\text { recA1, endA1, gyrA96, thi-1, hsdR17 ( } \mathrm{r} \\
\text { relA1, } \Delta(\text { lac-proAB)/F' }[\text { traD36, proAB }\end{array}$ & 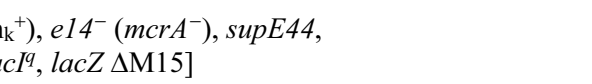 & 18 \\
\hline S17-1 & MM294 derivative, RP4-2 Tc::Mu-Km & 7 chromosomally integrated & 23 \\
\hline \multicolumn{4}{|c|}{ 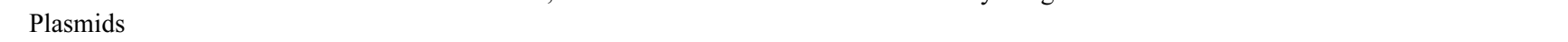 } \\
\hline pUCP18 & Escherichia-Pseudomonas shuttle vect & & 21 \\
\hline pFLCP01 & pUCP18 with a $2.2 \mathrm{~kb}$ PCR fragment $\mathrm{c}$ & ining $c t a B$ (Pf101_0124); $\mathrm{Cb}^{\mathrm{r}}$ & This study \\
\hline pFLCP02 & pUCP18 with a $2.1 \mathrm{~kb}$ PCR fragment $\mathrm{c}$ & ining $c t a C$ (Pf101_0354); $\mathrm{Cb}^{\mathrm{r}}$ & This study \\
\hline pFLCP03 & pUCP18 with a $2.2 \mathrm{~kb}$ PCR fragment $\mathrm{c}$ & ining ctaA (Pfl01_4431); $\mathrm{Cb}^{\mathrm{r}}$ & This study \\
\hline pK18mobsacB & $\mathrm{Km}^{\mathrm{r}} \mathrm{pUC} 18$ derivative, $l a c Z \alpha, m o b$ site & & 19 \\
\hline pUGMF01 & $\begin{array}{l}\text { pK19mobsacB with a 1.2-kb PCR fragn } \\
\text { fragment downstream of } c t a B \text { (Pfl01 } 0\end{array}$ & $\begin{array}{l}\text { upstream of } c t a B \text { (Pfl01_0124) and a 1.1-kb PCR } \\
; \mathrm{Km}^{\mathrm{r}}\end{array}$ & This study \\
\hline pUGMF02 & $\begin{array}{l}\text { pK } 19 m o b s a c B \text { with a } 1.3-\mathrm{kb} \text { PCR frag } \\
\text { PCR fragment downstream of } c t a C \text { (Pf }\end{array}$ & $\begin{array}{l}\text { tupstream of } c t a C \text { (Pfl01_0354) and a 1.4-kb } \\
\text { 0354); } \mathrm{Km}^{\mathrm{r}}\end{array}$ & This study \\
\hline pUGMF03 & $\begin{array}{l}\text { pK19mobsacB with a 1.3-kb PCR fragn } \\
\text { fragment downstream of ctaA (Pfl01_4 }\end{array}$ & $\begin{array}{l}\text { upstream of ctaA (Pfl01_4431) and a 1.2-kb PCR } \\
; \mathrm{Km}^{\mathrm{r}}\end{array}$ & This study \\
\hline
\end{tabular}


Table 2. Sequence of PCR primers used in this study

\begin{tabular}{ll}
\hline Primer name & \multicolumn{1}{c}{ Sequence (5' to 3') } \\
\hline PFL01f & ACGTAGAATTCGATCTGCCAGAAATGCCGTCC \\
PFL01r & ACGTAGGATCCGAGGGCAATGGCTGAAGCTG \\
PFL02f & ACGTACTGCAGCCGAGGTTTTGGTCGTATAC \\
PFL02r & ACGTAGGATCCGTGGGGCGAAAAACAGCAG \\
PFL03f & ACGTAGGATCCAGCTAAAGGTGACAGATGCGAC \\
PFL03r & ACGTACTGCAGGCGTTGATCTCCCTTGGTGAC \\
DPFL01Uf & ACGTACTGCAGGTAATCAGATCCACGCTGCTG \\
DPFL01Ur & ACGTAGGATCCTCTGACGGCTGCTCTACTTTGG \\
DPFL01Df & ACGTAGGATCCGCTTCAAGATCTGATCGCTTG \\
DPFL01Dr & ACGTAGAATTCATCTACATCCGCAACGGTAC \\
DPFL02Uf & ACGTAGTCGACTGCGCTGCTGTTATTTCGAC \\
DPFL02Ur & ACGTAGGATCCATGGCAACGATCCGTATCTG \\
DPFL02Df & ACGTAGCATGCGAACGAATACCTGAGCCAGAAC \\
DPFL02Dr & ACGTAGTCGACACCTTCAGGTCGATGTATCACG \\
DPFL03Uf & ACGTAAAGCTTTTGTCATAACGGCCCTTGAACG \\
DPFL03Ur & ACGTACTCGAGCAGTCGCATCTGTCACCTTTAG \\
DPFL03Df & ACGTACTCGAGTCCGACGAAGACGACCAACATC \\
DPFL03Dr & ACGTAGAATTCCGCTCGTGAGATTCAGCCATTG \\
DcheAUf & AGATCTGCAGTGACGCTAAAGATCACGAAGTTGC \\
DcheAUr & ACTAGAATTCGTTCGGACAGTTGCTCAAGAATCTC \\
DcheADf & CATAGAATTCGTCAGGAAGAATGGTCATCAAGC \\
DcheADr & GCATTCTAGACGATAAGGCTTAGAACATCCATCAG \\
\hline
\end{tabular}

PFL01r，PFL02f/PFL02r, and PFL03f/PFL03r primer sets, and cloned into broad-host-range plasmid pUCP18 (21) to construct pFLCP01, pFLCP02, and pFLCP03, respectively. Suicide plasmids pUGMF01, pUGMF02, and pUGMF03 were constructed for unmarked gene deletion in $P$. fluorescens Pf0-1. PCR using primer sets DPFL01Uf/DPFL01Ur and DPFL01Df/DPFL01Dr was conducted to amplify $1.2-\mathrm{kb}$ regions upstream and downstream of Pfl01 0124 from the $P$. fluorescens Pf0-1 genome, respectively. Amplified upstream and downstream regions were digested with Pst I-BamHI and BamHI-EcoRI, respectively, and ligated with the backbone of Pst I-EcoRI-digested pK18mobsacB (19) to obtain pUGMF01. PCR using primer sets DPFL02Uf/DPFL02Ur and DPFL02Df/DPFL02Dr was conducted to amplify a 1.4-kb upstream region and a 1.3-kb downstream region of Pfl01_0354 from the $P$. fluorescens Pf0-1 genome, respectively. Amplified upstream and downstream regions were digested with SalI-BamHI and SphI-SalI, respectively, and ligated with the backbone of SphI-BamHI-digested pK18mobsacB to obtain pUGMF02. PCR using primer sets DPFL03Uf/DPFL03Ur and DPFL03Df/DPFL03Dr was conducted to amplify a $1.3-\mathrm{kb}$ upstream region and a $1.2-\mathrm{kb}$ downstream region of Pfl01 0354 from the P. fluorescens Pf0-1 genome, respectively. Amplified upstream and downstream regions were digested with HindIII-XhoI and XhoI-EcoRI, respectively, and ligated with the backbone of HindIII-EcoRI-digested pK18mobsacB to obtain pUGMF03. The chromosomal Pf101 0124, Pf101 0354, and Pfl01 4431 genes were deleted by the unmarked gene deletion technique (19) using suicide plasmids pUGMF01, pUGMF02, and pUGMF03, respectively. Unmarked gene deletion was confirmed by PCR using PCR primers specific to upstream and downstream sites of each gene.

\section{Preparation of tomato root exudate}

Exudate was prepared from a plant species, tomato (Solanum lycopersicum cv. Oogatahukuju). Tomato seeds were sterilized by gentle shaking for $10 \mathrm{~min}$ in a solution of $8.75 \%(\mathrm{v} / \mathrm{v})$ sodium hypochloride supplemented with $0.1 \%(\mathrm{v} / \mathrm{v})$ Tween 20 . The sterilized seeds were soaked six times for $15 \mathrm{~min}$ in sterile demineralized water. Nine sterile seeds were placed in $3 \mathrm{~mL}$ SSE medium (2), consisting of $5 \mu \mathrm{M} \mathrm{KK_{2 } \mathrm { PO } _ { 4 } , 4 \mathrm { mM } \mathrm { CaSO }}, 2 \mathrm{mM}$ $\mathrm{MgCl}_{2}, 2.5 \mathrm{mM} \mathrm{NH}_{4} \mathrm{NO}_{3}, 0.5 \mathrm{mM} \mathrm{KOH}, 2.5 \mathrm{mM} \mathrm{NaOH}$, and 0.02 $\mathrm{mM} \mathrm{Fe}$ (as FeEDTA), and were allowed to grow in a climate- controlled growth chamber (NK System, Osaka, Japan) at $28^{\circ} \mathrm{C}$, and $16 \mathrm{~h}$ of daylight. After 18 days, root exudates were collected and evaporated to dryness at $45^{\circ} \mathrm{C}$ under a vacuum, dissolved in 1 $\mathrm{mL}$ water, and sterilized by membrane filtration $(0.45-\mu \mathrm{m}$ pore size $)$.

\section{Selection for rifampicin resistance mutants}

Spontaneous rifampicin-resistant mutants of $P$. fluorescens were generated by spreading bacterial cells, grown overnight in $2 \times \mathrm{YT}$, onto $2 \times$ YT agar plates containing $20 \mu \mathrm{g} \mathrm{mL}^{-1}$ rifampicin. The plates were incubated at $28^{\circ} \mathrm{C}$ for $20 \mathrm{~h}$ to form colonies. The resulting rifampicin-resistant colonies were then streaked on $2 \times$ YT agar containing $50 \mu \mathrm{g} \mathrm{mL}^{-1}$ rifampicin, and Rif ${ }^{\mathrm{f}}$ strains were subsequently maintained on this medium. One mutant showing a growth rate similar to that of the wild-type strain was selected and designated Pf0-1Rif. Similarly, a rifampicin-resistant mutant of $P$. fluorescens FLD3 was obtained and designated FLD3Rif.

\section{Gnotobiotic root colonization assays}

Forty grams of quartz sand $(0.1$ to $0.3 \mathrm{~mm})$ was placed in glass tubes (22 $\mathrm{mm}$ inner diameter, $25 \mathrm{~mm}$ outer diameter, $12 \mathrm{~cm}$ length) and compacted by gentle shaking. The open end of the tube was plugged with a silicone resin stopper. The tube was then autoclaved for $15 \mathrm{~min}$ at $121^{\circ} \mathrm{C}$. Ten milliliters of PNS (plant nutrient solution) (8), consisting of $1.25 \mathrm{mM} \mathrm{Ca}\left(\mathrm{NO}_{3}\right)_{2}, 1.25 \mathrm{mM} \mathrm{KNO}_{3}, 0.5 \mathrm{mM}$ $\mathrm{MgSO}_{4}, 0.25 \mathrm{mM} \mathrm{KH}_{2} \mathrm{PO}_{4}$, and trace elements (in $\mathrm{mg} \mathrm{L}^{-1}$ ): $\mathrm{Fe}$ (as FeEDTA), 4.6; B, 0.5; $\mathrm{Zn}, 0.05 ; \mathrm{Cu}, 0.02 ; \mathrm{Mo}, 0.01$, was added to an autoclaved sand column. Tomato seeds (S. lycopersicum $\mathrm{cv}$. Oogatahukuju) were sterilized as described at "Preparation of tomato root exudate" section. To synchronize germination, seeds were placed on Petri dishes containing PNS solidified with $1.5 \%$ (w/v) Bacto Agar (Becton, Dickinson and Company, Franklin Lakes, NJ, USA) and incubated overnight in the dark at $4^{\circ} \mathrm{C}$, followed by incubation at $28^{\circ} \mathrm{C}$ for 2 days. A germinated seed was aseptically placed at the center of a growth tube and $5 \mathrm{~mm}$ below the surface of quartz sand. Bacterial cells were grown for $14 \mathrm{~h}$ in $2 \times$ YT medium, centrifuged $(3,300 \times g, 2 \mathrm{~min})$, washed three times with PNS, and adjusted to $10^{7} \mathrm{CFU} \mathrm{mL} \mathrm{mL}^{-1}$ in PNS. For the colonization assay, 100 $\mu \mathrm{L}$ bacterial cell suspensions were added to the edge of a plant growth tube. For the competitive colonization assay, $100 \mu \mathrm{L}$ of $1: 1$ $(\mathrm{v} / \mathrm{v})$ mixture of the tested strain and the competitor were mixed and inoculated at the edge of a plant growth tube. The plant growth tubes were incubated in a climate-controlled growth chamber $\left(28^{\circ} \mathrm{C}\right.$, $16 \mathrm{~h}$ daylight) to allow the plantlets to grow. After 7 days of growth in the plant growth tubes, the root systems of tomato were mostly unbranched. The root tip (1 to $2 \mathrm{~cm}$ length) was removed and shaken vigorously in the presence of adhering sand particles in $0.5 \mathrm{~mL}$ of PNS to remove bacteria. The bacterial suspension was diluted and $100 \mu \mathrm{L}$ was plated on $2 \times$ YT agar plates. For the competitive colonization assay, the bacterial suspension was spread on $2 \times$ YT agar plates with and without rifampicin. For statistical analysis, the nonparametric Wilcoxon-Mann-Whitney test was used (27).

\section{Results}

Chemotactic responses toward amino acids by $\mathrm{P}$. fluorescens PfO-1

Amino acids are one of the major tomato exudate components (25) and strong chemoattractants of $P$. fluorescens strains $(6,26,28)$; therefore, we focused the present study on the identification of a chemotaxis sensory protein(s) for amino acids in $P$. fluorescens Pf0-1. We first measured chemotactic responses of $P$. fluorescens Pf0-1 toward each of twenty commonly-occurring L-amino acids by the computer-assisted capillary assay (16). P. fluorescens Pf0-1 exhibited chemotactic responses toward all twenty amino acids (Table 3). In particular, Cys, Gln, Gly, Ile, Lys, 
Table 3. Chemotactic responses of $P$. fluorescens Pf0-1 wild-type and mutant strains toward amino acids

\begin{tabular}{|c|c|c|c|c|c|}
\hline \multirow{2}{*}{ Compounds } & \multicolumn{5}{|c|}{ Chemotactic responses* } \\
\hline & Pf0-1 & FL0124 ( $\Delta c t a A c t a C)$ & FL0354 ( $\triangle c t a A c t a B)$ & FL4431 $(\Delta c t a B c t a C)$ & FLD3 ( $\triangle c t a A c t a B c t a C)$ \\
\hline Ala & ++ & + & - & + & - \\
\hline Arg & ++ & +++ & + & ++ & - \\
\hline Asn & ++ & ++ & - & +++ & - \\
\hline Asp & ++ & - & - & - & - \\
\hline Cys & +++ & +++ & +++ & +++ & ++ \\
\hline Gln & +++ & ++ & - & - & - \\
\hline Glu & + & ++ & - & - & - \\
\hline Gly & +++ & ++ & + & + & - \\
\hline His & ++ & +++ & - & ++ & - \\
\hline Ile & +++ & +++ & - & +++ & - \\
\hline Leu & ++ & - & - & +++ & - \\
\hline Lys & +++ & ++ & - & ++ & - \\
\hline Met & +++ & +++ & +++ & +++ & - \\
\hline Ser & +++ & ++ & - & +++ & - \\
\hline Thr & ++ & +++ & + & +++ & - \\
\hline Trp & + & - & - & - & - \\
\hline Tyr & ++ & + & - & + & - \\
\hline Val & ++ & +++ & - & +++ & - \\
\hline
\end{tabular}

* Videotape frames were analyzed at the initiation of observation and 1 min after the initiation. Normalized cell numbers were calculated by dividing the number of bacteria at $1 \mathrm{~min}$ by that at the initiation of the observation. The strength of chemotaxis is presented as the normalized cell number: $+++>3 ;++\leq 3->2 ;+\leq 2->1.5 ;-$, no response.

Met, Phe, Pro, and Ser were strong chemoattractants of $P$. fluorescens Pf0-1, while it showed weak chemotactic responses toward Glu and Trp.

\section{Identification of chemotaxis sensory proteins for amino acids}

In Pseudomonas aeruginosa PAO1, PctA, PctB, and PctC have been identified as MCPs for amino acids $(12,31)$. There is $46-70 \%$ identity among amino acid sequences of periplasmic domains of these MCPs. To search for the PctA homologue of $P$. fluorescens Pf0-1, BLASTP analysis was performed on a protein database of $P$. fluorescens Pf0-1 by using the 250 amino acid sequence of the putative periplasmic domain of PctA (residues 28 to 277 of PctA [accession number: NP 252999.1]) as a query sequence. BLASTP search found that three proteins, Pf101_4431 (accession number: ABA76168), Pf101_0124 (ABĀ71868), and Pf101_0354 (ABA72098), showed the highest similarity to the query sequence $(65 \%, 58 \%$, and $46 \%$ identity, respectively). They possessed $\mathrm{HCD}$ in the $\mathrm{C}$-terminal regions and have been annotated as MCPs on the basis of homology. Pf101_4431, Pf101_0124, and Pf101_0354 are 59, 56, and 44\% identical to the periplasmic domain of PctB (residues 27 to 277), and 49,45 , and $51 \%$ identical to that of PctC (residues 27 to 280).

To investigate whether Pf101_4431, Pf101_0124, and Pf101_0354 act as MCPs for amino acids, their genes were clone $\bar{d}$ into broad-host-range plasmid pUCP18 (21), the resulting recombinant plasmids were introduced into the $p c t A$ pctB pctC triple mutant of $P$. aeruginosa PAO1 ( $P$. aeruginosa PCT2) (31), and the recombinant strains were examined for chemotactic responses to amino acids. Whereas $P$. aeruginosa PCT2 was non-chemotactic to all amino acids tested, PCT2 (pFLCP01[containing the Pf101_0124 gene]), PCT2 (pFLCP02 [containing the Pfl01_0354 gene]), and PCT2 (pFLCP03 [containing the Pfl01_4431 gene]) showed
Table 4. Chemotactic responses of $P$. aeruginosa PCT2 containing $P$. fluorescens PfO- 1 ctaA, ctaB, or ctaC genes toward amino acids

\begin{tabular}{lccc}
\hline & \multicolumn{3}{c}{ Chemotactic responses* } \\
\cline { 2 - 4 } Compounds & PCT2 (pFLCP01) & PCT2 $($ pFLCP02) & PCT2 (pFLC03) \\
& $(+c t a B)$ & $(+$ ctaC $)$ & $(+c t a A)$ \\
\hline Ala & +++ & - & +++ \\
Arg & + & - & +++ \\
Asn & +++ & - & + \\
Asp & ++ & - & ++ \\
Cys & - & - & +++ \\
Gln & ++ & - & - \\
Glu & + & - & - \\
Gly & + & - & +++ \\
His & - & - & +++ \\
Ile & - & - & +++ \\
Leu & ++ & - & +++ \\
Lys & +++ & - & +++ \\
Met & +++ & ++ & +++ \\
Phe & + & - & ++ \\
Pro & + & - & +++ \\
Ser & +++ & - & +++ \\
Thr & + & - & +++ \\
Trp & - & - & +++ \\
Tyr & + & - & ++ \\
Val & ++ & - & + \\
\hline
\end{tabular}

* Chemotactic responses are the same as in Table 3.

chemotactic responses to 16,1 , and 18 amino acids, respectively (Table 4), demonstrating that these proteins act as MCPs for amino acids. PCT2 (pFLCP01) and PCT2 (pFLCP03) showed strong responses to 5 amino acids (Ala, Asn, Lys, Met, and Ser) and 14 amino acids (Ala, Arg, Cys, Gly, His, Ile, Leu, Lys, Met, Pro, Ser, Thr, Trp, and Val), 


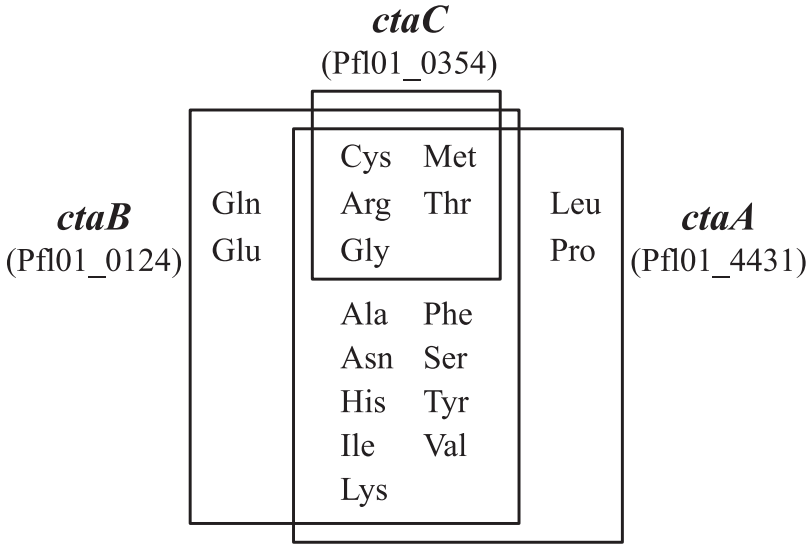

Fig. 1. Representation of 20 commonly-occurring L-amino acids detected by chemotaxis sensory proteins. This Venn diagram is constructed based on chemotactic responses by FL0124, FL0354, and FL4431 (Table 3).

respectively, while PCT2 (pFLCP02) exhibited moderate responses only to Met. No common structural features are found among side chains of amino acids to which both PCT (pFLCP01) and PCT2 (pFLCP03) responded. Based on these results, the Pf101_4431, Pf101_0124, and Pf101_0354 genes were designated ctaA, ctaB, and ctaC (cta: chemotactic transducer of amino acids).

We constructed a ctaA ctaB ctaC triple mutant of $P$. fluorescens Pf0-1 (P. fluorescens FLD3) to assess the possibility of chemotaxis sensory protein(s) other than CtaA, $\mathrm{CtaB}$, and $\mathrm{CtaC}$. The triple mutant FLD3 showed moderate responses only toward Cys and Pro (Table 3). We then examined $c t a B c t a C, c t a A c t a C$, and ctaA ctaB double mutants of $P$. fluorescens Pf0-1 (P. fluorescens FL4431, FL0124, and FL0354, respectively) for their chemotaxis to amino acids to investigate the role of each MCP in amino acid chemotaxis in $P$. fluorescens $\mathrm{Pf0}-1$. The ctaA ctaB double mutant showed a strong response to Met and Cys and weak or moderate responses to Arg, Gly, Pro, and Thr (Table 3). Since $P$. fluoresces FLD3 showed moderate responses to Cys and Pro, Met is the main chemoattractant of $\mathrm{CtaC}$. The $\operatorname{ctaB} \operatorname{ctaC}$ and ctaA ctaC double mutants showed strong responses to several amino acids and their response patterns were similar to those of PCT2 (pFLCP03) and PCT2 (pFLCP01), respectively. These results suggest that $\mathrm{CtaA}$ and $\mathrm{CtaB}$ play the major roles in amino acid chemotaxis in P. fluorescens Pf0-1 (Fig. $1)$.

Of the major root exudate components other than amino acids, malic acid and succinic acid were strong attractants to P. fluorescens Pf0-1 (data not shown); therefore, we examined $P$. fluorescens FLD3 for its ability to respond to malic acid and succinic acid. It showed chemotactic responses to malic acid and succinic acid comparable to those by the parental strain (data not shown), suggesting that $\mathrm{CtaA}, \mathrm{CtaB}$, and $\mathrm{CtaC}$ are not involved in the detection of malic acid and succinic acid.

\section{Chemotaxis of $\mathrm{P}$. fluorescens strains to tomato root exudate}

$P$. fluorescens Pf0-1 wild-type and mutant strains were tested for chemotaxis to tomato root exudate to assess the involvement of MCPs for amino acids in chemotaxis to the

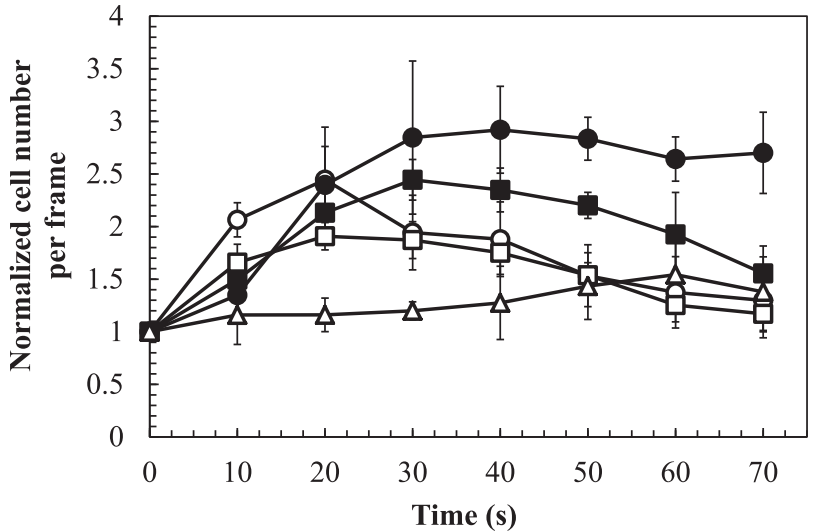

Fig. 2. Chemotactic responses to tomato root exudate by $P$. fluorescens Pf0-1 (closed circles), FL0124 (open circles), FL0354 (open squares), FL4431 (closed squares), and FLD3 (open triangles). Digital image processing was used to count the number of bacteria around the mouth of a capillary containing exudates and $1 \%(\mathrm{w} / \mathrm{v})$ agarose. One videotape frame was analyzed at each time point. The chemotactic response is presented as the normalized cell number. The normalized cell number was calculated by dividing the number of bacteria at each time point by that at the initiation of the observation. Vertical bars represent the standard deviations of measurements done in triplicate experiments.

root exudates. P. fluorescens Pf0-1 wild-type strain was strongly attracted by tomato root exudate, while $\operatorname{ctaA} \operatorname{ctaB}$ ctaC triple mutant showed much decreased responses (Fig. 2 ). The double mutants showed stronger responses to root exudate than the triple mutant, but weaker responses than the $P$. fluorescens Pf0-1 wild-type strain. In particular, ctaA ctaB double mutant showed the weakest responses among the double mutants. This result suggests that amino acids are the major chemoattractants of $P$. fluorescens Pf0-1 in tomato root exudate. It also suggests that $\mathrm{CtaA}, \mathrm{CtaB}$, and $\mathrm{CtaC}$ are responsible for chemotaxis to root exudate to various degrees and that $\mathrm{CtaC}$ is less responsible than $\mathrm{CtaA}$ and $\mathrm{CtaB}$.

\section{Root colonization analysis}

In order to investigate the importance of chemotaxis to amino acids in the root colonization process, we examined $P$. fluorescens $\mathrm{Pf0}$-1, spontaneous rifampicin resistant mutant of Pf0-1 (Pf0-1Rif), the ctaA ctaB ctaC triple mutant, and its spontaneous rifampicin-resistant mutant (FLD3Rif) as well as double mutants for their root-colonizing ability by the gnotobiotic root colonization system. We also tested the cheA mutant of Pf0-1, which is a general non-chemotactic mutant, with the root colonization assay to confirm the report by de Weert et al. that flagella-driven chemotaxis is an important trait for tomato root colonization by P. fluorescens (6). We confirmed that there were no significant differences in growth in LB medium between mutants and the wild type Pf0-1. When germinated tomato seedlings were inoculated with single strains, all mutants colonized the tomato root to the same extent as the wild-type strain (Fig. 3A). We then carried out competitiveness assays between chemotaxis mutants and the wild-type strain by inoculating seedlings with a $1: 1$ mixture. Because Pf0-1Rif and FLD3Rif competed fully with Pf0-1 and FLD3, respectively (data not shown), we used Pf01Rif and FLD3Rif as competitor strains in competitive colonization assays to distinguish the competing strains from 

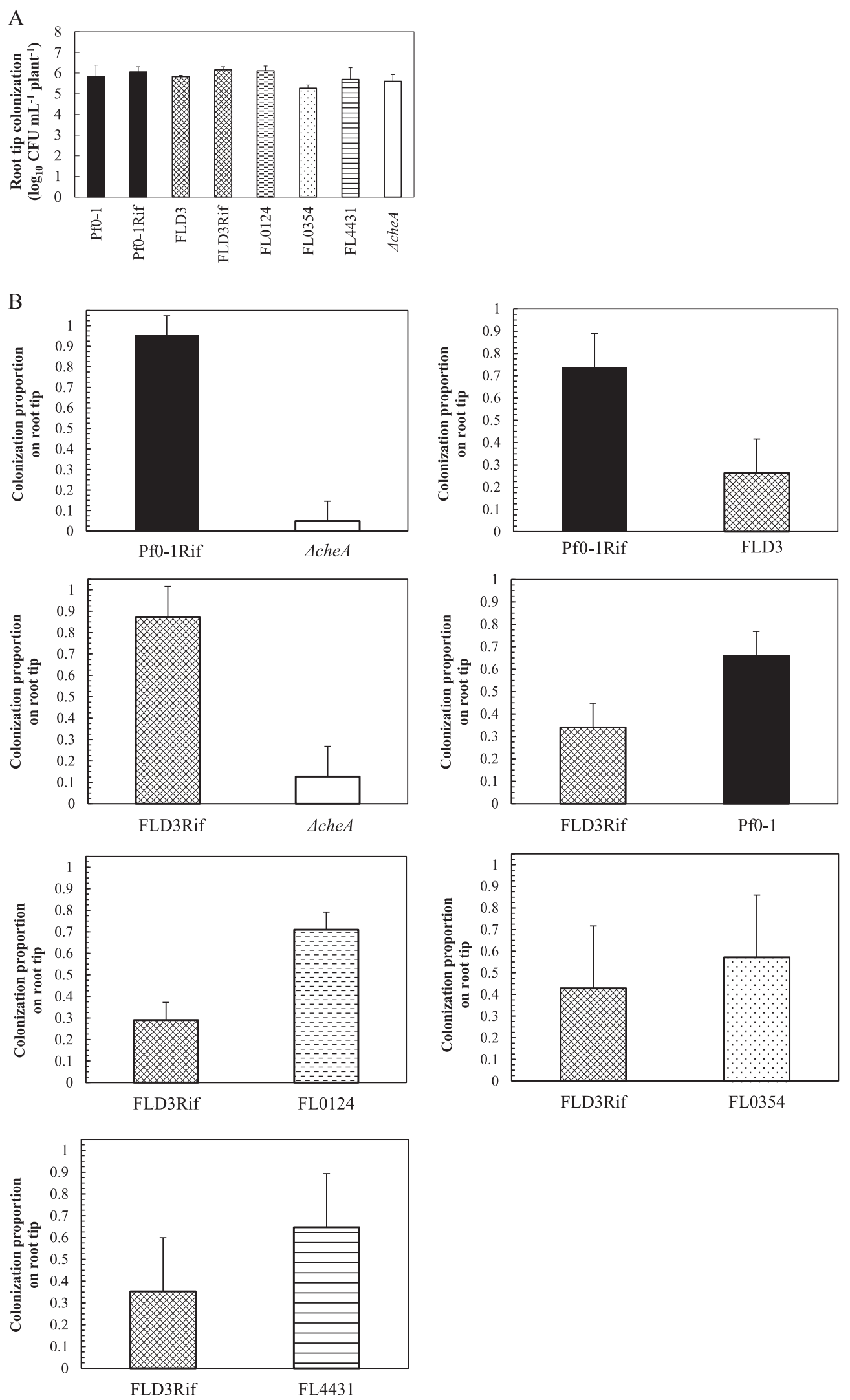

Fig. 3. Tomato root tip colonization by $P$. fluorescens strains, (A) alone and (B) in competition with Rif ${ }^{\mathrm{r}}$ mutants. After 7 days, root systems were sampled. Bar indicates standard deviation. The total CFU numbers at the root tip in Fig. 3B were the same level as in Fig. 3A. There were significant $(P<0.05)$ differences in colonization between Pf0-1Rif and $\triangle c h e A$, Pf0-1Rif and FLD3, FLD3Rif and $\triangle c h e A$, FLD3Rif and Pf0-1, and FLD3Rif and FL0124. 
tested strains. As previously shown by de Weert et al. (6), the non-chemotactic cheA mutant was a very poor competitor and showed more than 10-fold reduced ability to colonize tomato roots (Fig. 3B). The ctaA ctaB ctaC triple mutant exhibited higher competitive colonization ability than the cheA mutant, but it still showed an approximately 2-fold impaired colonization ability in the competitive colonization assay. This result indicates that chemotaxis to amino acids plays a role in the root colonization process by $P$. fluorescens. FL0124 (ctaA ctaC double mutant) and FL4431 (ctaB ctaC double mutant) showed almost 2-fold superior colonization ability than FLD3Rif, while FL0354 (ctaA ctaB) showed only 1.3-fold superior colonization ability than FLD3Rif. We then examined competitiveness between $\triangle c h e A$ (cheA mutant) and FLD3Rif (ctaA ctaB ctaC triple mutant). As shown in Fig. $3 \mathrm{~B}$, FLD3Rif is more competitive than che A mutant.

\section{Discussion}

There are two classes of MCPs for amino acids in bacteria. One class includes Tar and Tsr of E. coli and Salmonella enterica serovar Thyphimurium, and the other includes $P$. aeruginosa PAO1 PctA. Tsr is an MCP for the attractants Ser, Ala and Gly, while Tar is an MCP for attractants Asp and Glu (29). These MCPs possess short periplasmic domains (ca. 150 amino acid residues), and their ligand specificity is relatively narrow. $P$. aeruginosa PAO1 PctA, which detects 18 commonly-occurring L-amino acids, shows broader ligand specificity, and its periplasmic domain (ca. 240 amino acid residues) is longer than those of Tsr and Tar (12). CtaA and $\mathrm{CtaB}$, the main MCPs for amino acids in P. fluorescens Pf01, detect 16 amino acids (Fig. 1, Table 3), and possess long periplasmic domains, suggesting that $\mathrm{CtaA}$ and $\mathrm{CtaB}$ belong to the class of PctA-type MCPs. BLASTP search of genome databases revealed that like $P$. aeruginosa PAO1 and $P$. fluorescens Pf0-1, other Pseudomonas bacteria possess 2-4 PctA-type MCPs, but not Tar/Tsr homologues. Thus, amino acids are supposed to be major chemoattractants of Pseudomonas bacteria.

de Weert et al. reported that the cheA mutant of $P$. fluorescens WCS365 was much less competitive for tomato root colonization than the wild-type strain and concluded that flagella-driven chemotaxis toward root exudate is an important trait for competitive root colonization (6); however, it has not been identified which chemotaxis ligands in root exudate are involved in effective root colonization. In this study, we demonstrated that ctaA ctaB ctaC mutant impaired in chemotaxis to amino acids showed a significant reduced ability to colonize tomato root in competitive root colonization assays using the wild-type strain as the competitor strain (Fig. 3B). This triple mutant was impaired in chemotaxis to amino acids (Table 3), but showed parental responses to malic acid and succinic acid, another major components of root exudate. These results suggest that amino acids play a role as chemoattractants for effective root colonization. Data in Table 3 and Fig. 1 suggest that $\mathrm{CtaA}$ and $\mathrm{CtaB}$ are the major MCPs for amino acids and root exudate and that $\mathrm{CtaC}$ contributes less to chemotaxis to amino acids and root exudate. Consistent with these data, $\mathrm{CtaA}$ and $\mathrm{CtaB}$ also make a greater contribution to the root colonization process than $\mathrm{CtaC}$ (Fig. 3B). In this study, we demonstrate that chemotaxis to amino acids is involved in effective root colonization by $P$. fluorescens, but it remains to be elucidated which amino acids are involved in this process.

Additionally, ctaABC mutant was more competitive for root colonization than cheA mutant (Fig. 3B), suggesting that chemoattractants other than amino acids are involved in root colonization. Since P. fluorescens Pf0-1 exhibits marked responses to organic acids (especially succinic acid and malic acid) (data not shown), we suppose that chemotaxis to organic acids such as succinic acid and malic acid is also involved in root colonization by $P$. fluorescens. We are now searching MCPs for organic acids in P. fluorescens Pf0-1 to investigate the involvement of chemotaxis to organic acids in root colonization.

\section{References}

1. Aarons, S., A. Abbas, C. Adams, A. Fenton, and F. O'Gara. 2000. A regulatory RNA (Prrb RNA) modulates expression of secondary metabolite genes in Pseudomonas fluorescens F113. J. Bacteriol. 182:3913-3919.

2. Angle, J.S., S.P. Mcgrath, and R.L. Chaney. 1991. New culture medium containing ionic concentrations of nutrients similar to concentrations found in the soil solution. Appl. Environ. Microbiol. 57:3674-3676.

3. Barahona, E., A. Navazo, F. Martínez-Granero, T. Zea-Bonilla, R.M. Pérez-Jiménez, M. Martín, and R. Rivilla. 2011. Pseudomonas fluorescens F113 mutant with enhanced competitive colonization ability and improved biocontrol activity against fungal root pathogens. Appl. Environ. Microbiol. 77:5412-5419.

4. Bolwerk, A., A.L. Lagopodi, A.H. Wijfjes, G.E. Lamers, T.F. ChinA-Woeng, B.J. Lugtenberg, and G.V. Bloemberg. 2003. Interactions in the tomato rhizosphere of two Pseudomonas biocontrol strains with the phytopathogenic fungus Fusarium oxysporum f. sp. radicislycopersici. Mol. Plant Microbe Interact. 16:983-993.

5. Compeau, G., B.J. Al-Achi, E. Platsouka, and S.B. Levy. 1988. Survival of rifampin-resistant mutants of Pseudomonas fluorescens and Pseudomonas putida in soil systems. Appl. Environ. Microbiol. 54:2432-2438.

6. de Weert, S., H. Vermeiren, I.H. Mulders, I. Kuiper, N. Hendrickx, G.V. Bloemberg, J. Vanderleyden, R. de Mot, and B.J. Lugtenberg. 2002. Flagella-driven chemotaxis towards exudate components is an important trait for tomato root colonization by Pseudomonas fluorescens. Mol. Plant Microbe Interact. 15:1173-1180.

7. Falke, J.J., and G.L. Haselbauer. 2001. Transmembrane signaling in bacterial chemoreceptors. Trends Biochem. Sci. 26:257-265.

8. Hoffland, E., G.R. Findenegg, and J.A. Nelemans. 1989. Solubilization of rock phosphate by rape. Plant Soil. 113:161-165.

9. Holloway, B.W., V. Krishnapillai, and A.F. Morgan. 1979. Chromosomal genetics of Pseudomonas. Microbiol. Rev. 43:73-102.

10. Kamilova, F., L.V. Kravchenko, A.I. Shaposhnikov, T. Azarova, N. Makarova, and B. Lugtenberg. 2006. Organic acids, sugars, and Ltryptophane in exudates of vegetables growing on stonewool and their effects on activities of rhizosphere bacteria. Mol. Plant Microbe Interact. 19:250-256.

11. Kato, J. 2008. Pseudomonas motility and chemotaxis, p. 109-128. In B.H.A. Rem (ed.), Pseudomonas. Wiley-VCH, Weinheim.

12. Kuroda, A., T. Kumano, K. Taguchi, T. Nikata, J. Kato, and H. Ohtake. 1995. Molecular cloning and characterization of a chemotactic transducer gene in Pseudomonas aeruginosa. J. Bacteriol. 177:7019-7025.

13. Lugtenberg, B.J.J., L.A. de Weger, and J.W. Bennett. 1991. Microbial stimulation of plant growth and protection from disease. Curr. Opin. Biotechnol. 2:457-464.

14. Lugtenberg, B.J.J., L.V. Kravchenko, and M. Simons. 1999. Tomato seed and root exudate sugars: composition, utilization by Pseudomonas biocontrol strains and role in rhizosphere colonization. Environ. Microbiol. 1:439-446. 
15. Masduki, A., J. Nakamura, T. Ohga, R. Umezaki, J. Kato, and H. Ohtake. 1995. Isolation and characterization of chemotaxis mutants and genes of Pseudomonas aeruginosa. J. Bacteriol. 177:948-952.

16. Nikata, T., K. Sumida, J. Kato, and H. Ohtake. 1992. Rapid method for analyzing bacterial behavioral responses to chemical stimuli. Appl. Environ. Microbiol. 58:2250-2254.

17. O'Sullivan, D.J., and F.O'Gara. 1992. Traits of fluorescent Pseudomonas spp. involved in suppression of plant root pathogens. Microbiol. Rev. 56:662-676.

18. Sambrook, J., E.F. Fritsch, and T. Maniatis. 1989. Molecular Cloning: a Laboratory Manual, 2nd ed. Cold Spring Harbor Laboratory, Cold Spring Harbor, NY.

19. Schäfer, A., A. Tauch, W. Jäger, J. Kalinowski, G. Thierbach, and A Pühler. 1994. Small mobilizable multi-purpose cloning vectors derived from the Escherichia coli plasmids pK18 and pK19: selection of defined deletions in the chromosome of Corynebacterium glutamicum. Gene 145:69-73.

20. Scher, F.M., J.W. Kloepper, and C.A. Singleton. 1985. Chemotaxis of fluorescent Pseudomonas spp. to soybean seed exudates in vitro and in soil. Can. J. Microbiol. 31:570-574.

21. Schweizer, H.P. 1991. Escherichia-Pseudomonas shuttle vectors derived from pUC18/19. Gene 97:109-121.

22. Shanahan, P., A. Borro, F.O'Gara, and J.D. Glennon. 1992. Isolation, trace enrichment and liquid chromatographic analysis of diacetylphloroglucinol in culture and soil samples using UV and amperometric detection. J. Chromatogr. 606:171-177.

23. Simon, R., U. Prifer, and A. Pühler. 1983. A broad host range mobilization system for in vivo genetic engineering: transposon mutagenesis in Gram negative bacteria. Nat. Biotechnol. 1:784-791.

24. Simons, M., A.J. van der Bij, I. Brand, L.A. de Weger, C.A. Wijffelman, and B.J. Lugtenberg. 1996. Gnotobiotic system for studying rhizosphere colonization by plant growth-promoting Pseudomonas bacteria. Mol. Plant Microbe Interact. 9:600-607.
25. Simons, M., H.P. Permentier, L.A. de Weger, C.A. Wijffelman, and B.J.J. Lugtenberg. 1997. Amino acid sysnthesis is necessary for tomato root colonization by Pseudomonas fluorescens strain WCS365. Mol. Plant Microbe Interact. 10:102-106.

26. Singh, T., and D.K. Arora. 2001. Motility and chemotactic response of Pseudomonas fluorescens toward chemoattractants present in the exudate of Macrophomina phaseolina. Microbiol. Res. 156:343-351.

27. Sokal, R.R., and F.J. Rohlf. 1981. Biometry. Freeman and Co., San Francisco.

28. Sood, S.G. 2003. Chemotactic response of plant-growth-promoting bacteria towards roots of vascular-arbuscular mycorrhizal tomato plants. FEMS Microbiol. Ecol. 45:219-227.

29. Stewart, R.C., and F.W. Dahlquist. 1987. Molecular components of bacterial chemotaxis. Chem. Rev. 87:997-1025.

30. Suslow, T.V. 1982. Role of root-colonizing bacteria in plant growth, p. 187-223. In M.S. Mount, and G.H. Lacy (ed.), Phytopathogenic Prokaryotes, Voll. Academic Press, London.

31. Taguchi, K., H. Fukutomi, A. Kuroda, J. Kato, and H. Ohtake. 1997. Genetic identification of chemotactic transducers for amino acids in Pseudomonas aeruginosa. Microbiology 143:3223-3229.

32. Thomashow, L.S., and D.M. Weller. 1988. Role of a phenazine antibiotic from Pseudomonas fluorescens in biological control of Gaeumannomyces graminis var. tritici. J. Bacteriol. 170:3499-3508.

33. Thomashow, L.S., D.M. Weller, R.F. Bonsall, and L.S. Pierson. 1990. Production of the antibiotic phenazine-1-carboxylic acid by fluorescent Pseudomonas species in the rhizosphere of wheat. Appl. Environ. Microbiol. 56:908-912.

34. Zhulin, I.B. 2001. The superfamily of chemotaxis transducers: from physiology to genomics and back. Adv. Microb. Physiol. 45:157-198. 